\title{
LESSON 140
}

Letter 48

PAPER: A4.

Address a suitable envelope.
To-day's date

To: A. Cooke, Eg., Paddock Lane Comprehensive School, Paddock Lave Genstone. STH IGS

or Dt. Mrs Cooke, 4 may tembet th. When we mets rectly, $y$ mentioned ts need $f$ a book which canbines typeuritg o Office

or Practice, I am enitg to tell $y$ that lve shall be shoobly be Publishg: Evans IIT TERMEBIATE SECRETARIAL PRACTICE, a

N.P. book of projects wh. Combines these Subjects. [A quastionnaive sents by Mrs. Evans to employers o former students who had bn employed for a shat time showed toh, although shudents had done very wll in / exaninaths, they were findg / transith fo

OFFICE Simulated offurce coork to / real thing difficlt, nots least bec all work must be pefect when piesented to / boss, who does not give marks out of 10 but demands that ensatisfactory

N.P. work be retyped! [SECRETARIAL PRACTICE, bhorefote, is dasigned to give students / variety + realistic experience that theyneed to Rrepare them $f$ thair first iob:

(i) All / projects are based on / work of real offices.

(ii) Bec each unit is based on / work of a particular tind of fiom, I book offers experience of a great variety of office work.

Typist!

Display this section please!

(iii) The prijects ho bn designed to help shedents wrok on their oum w. a minimum of supervision: evors ho bn incleded so that stredents must learn to use common sense, 9 not always slairshly to follow / copy.

(iv) Each project involves / shedant in a variety of actirities, including such things as 1 use of reference sources 9 selection of methods of duplicaining.

(v) Most of there variety of handuriting styles.

(vi) Samples of letter headings or forms fot ea. Campany are printed at / back of / book fot / teacher to duplicate as requited.

N.P. We feel that sthis book wll help / teacher to plan more tealistic office Practice lessons 9 wll provide more enjoyable - Satisfactory lessons for / shudents. Ishall be very pleased to send $y$ an inspection copy if $y$ evish.

Sates Makageress 